\title{
SCATTERING OF WATER WAVES BY A SUBMERGED THIN VERTICAL WALL WITH A GAP
}

\author{
SUDESHNA BANERJEA ${ }^{\prime}$ and B. N. MANDAL ${ }^{2}$
}

(Received 15 February 1995; revised 12 March 1996)

\begin{abstract}
A train of surface water waves normally incident on a thin vertical wall completely submerged in deep water and having a gap, experiences reflection by the wall and transmission through the gaps above and in the wall. Using Havelock's expansion of water wave potential, two different integral equation formulations of the problem are presented. While the first formulation involves multiple integral equations which are solved here by reducing them to a singular integral equation with Cauchy kernel in a double interval, the second formulation involves a first-kind singular integral equation in a double interval with a combination of logarithmic and Cauchy kernel, the solution of which is obtained by utilizing the solution of a singular integral equation with Cauchy kernel in $(0, \infty)$ and also in a double interval. The reflection coefficient is evaluated by both the methods.
\end{abstract}

\section{Introduction}

Assuming linear theory, water wave scattering problems involving thin vertical barriers in deep water for a normally incident surface wave train are amongst the few which admit of exact solutions. Many researchers have contributed significantly to these problems by utilizing a variety of mathematical methods. Dean [3] first considered the problem of water-wave scattering by a thin vertical barrier completely submerged in deep water by using complex variable theory. Later Ursell [7] considered the complementary problem of a partially immersed thin vertical barrier and used an integral equation formulation based on Havelock's expansion of water wave potential to solve it. Evans [4] considered the submerged vertical plate problem and used complex variable theory in conjunction with Riemann-Hilbert boundary-value problem theory to solve it. Porter [6] investigated water wave scattering by a thin

\footnotetext{
${ }^{1}$ Department of Mathematics, Jadavpur University, Calcutta 700 032, INDIA

${ }^{2}$ Physics and Applied Mathematics Unit, Indian Statistical Institute, 203, B.T. Road, Calcutta 700035 , INDIA

(C) Australian Mathematical Society, 1998, Serial-fee code 0334-2700/98
} 
vertical wall with a submerged gap and used an integral equation formulation based on a suitable application of Green's integral theorem and also complex variable theory. The methods give rise to the same Riemann problem.

Recently Chakrabarti and Vijayabharati [2] considered the problem of water-wave transmission through a gap in a thin vertical wall completely submerged in deep water. They used complex variable theory to reduce the problem to two independent Riemann-Hilbert problems. In the present paper this problem is reinvestigated by using two different integral-equation formulations based on Havelock's expansion of the water wave potential. The first formulation involves multiple integral equations in the regions occupied by the wall and by the gaps above and in the wall. These multiple integral equations are reduced to a singular-integral equation with a Cauchy kernel in a double interval. The reflection coefficient is then evaluated. It agrees with the result obtained in [2] by a different method, except for the signs of a few terms.

In the second formulation, a singular integral equation in a double interval is obtained. Its kernel involves a combination of logarithmic and Cauchy-type singularities. It may be noted here that for the partially immersed barrier problem considered by Ursell [7], the same integral equation for a single infinite interval $(a, \infty)$ was obtained, $a$ being the depth to which the barrier is immersed, and because of this, it could be reduced to another singular integral equation with Cauchy kernel. However, in the present case such reduction is no longer possible, and as such it requires special attention. This integral equation is solved here by utilizing the solution of a singular integral equation with Cauchy kernel in $(0, \infty)$ and also in a double interval. The reflection coefficient is then evaluated. This result agrees with that obtained by the first approach.

\section{Formulation of the problem}

We consider irrotational motion of an incompressible inviscid fluid occupying the region $y \geq 0$, where the $y$-axis is taken vertically downwards into the fluid and the plane $y=0$ represents the undisturbed free surface. A train of time-harmonic surface waves with circular frequency $\sigma$ is incident from negative infinity on a thin vertical submerged wall with a gap. The wall occupies the position $x=0, y \in \mathscr{W}$ where $\mathscr{W}=(a, b) U(c, \infty)$ while the wall's gap has the position $x=0, b<y<c$. If the fluid motion is described by the velocity potential $\operatorname{Re}\left\{\phi(x, y) e^{-i \sigma t}\right\}$, then assuming linear theory, $\phi$ satisfies

$$
\begin{gathered}
\nabla^{2} \phi=0 \text { in } \quad y \geq 0, \\
K \phi+\phi_{y}=0 \quad \text { in } \quad y=0 .
\end{gathered}
$$


Here $K=\sigma^{2} / g, g$ being the acceleration due to gravity,

$$
\begin{gathered}
\phi_{x}=0 \text { on } x=0, y \in \mathscr{W}, \\
r^{1 / 2} \nabla \phi \text { is bounded as } r \rightarrow 0,
\end{gathered}
$$

where $r$ is the distance from the submerged edges of the wall, viz., the points $(0, a),(0, b)$ and $(0, c)$,

$$
\nabla \phi \rightarrow 0 \quad \text { as } \quad y \rightarrow \infty
$$

and

$$
\phi \rightarrow \begin{cases}T e^{-K y+i K z} & \text { as } \quad x \rightarrow \infty, \\ e^{-K y+i K x}+R e^{-K y-i K x} & \text { as } \quad x \rightarrow-\infty,\end{cases}
$$

where $T$ and $R$ are respectively the unknown transmission and reflection coefficients which will be determined in the course of solution. Here $e^{-K y+i K x}$ represents the velocity potential for the incident wave train.

The boundary-value problem represented by $(2.1)$ to (2.6) is now solved by two methods as described in the next section.

\section{Method of solution}

(a) Method based on reduction to multiple integral equations. By Havelock's expansion of water-wave potential a suitable representation of $\phi(x, y)$ satisfying $(2.1)$, (2.2), (2.5) and (2.6) is given by

$$
\begin{aligned}
& \phi(x, y)=T e^{-K y+i K x}+\int_{0}^{\infty} A(k) L(k, y) e^{-k x} d k \text { for } x>0, \\
& \phi(x, y)=e^{-K y+i K x}+R e^{-K y-i K x}+\int_{0}^{\infty} B(k) L(k, y) e^{k x} d k \text { for } x<0,
\end{aligned}
$$

where

$$
L(k, y)=k \cos k y-K \sin k y .
$$

The continuity of the horizontal velocity across $x=0$ for all $y>0$ produces, by Havelock's inversion theorem,

$$
T=1-R, \quad A(k)=-B(k) .
$$


Use of (3.1) in (2.3) produces

$$
\int_{0}^{\infty} k A(k) L(k, y) d k=i K T e^{-K y} \quad \text { for } \quad y \in \mathscr{W} .
$$

Again, the continuity of $\phi(x, y)$ across the gaps above and in the wall produces after using (3.3)

$$
\int_{0}^{\infty} A(k) L(k, y) d k=R e^{-k y} \quad \text { for } \quad y \in \mathscr{G},
$$

where $\mathscr{G}=(0, a) U(b, c)$. It may be noted that $\mathscr{G} U \mathscr{W}=(0, \infty)$. Application of the operator $\frac{d}{d y}+K$ to (3.4) and (3.5) gives rise to the multiple integral equations

$$
\begin{gathered}
\int_{0}^{\infty} k D(k) \sin k y d k=0 \text { for } \quad y \in \mathscr{W} \\
\int_{0}^{\infty} D(k) \sin k y d k=0 \text { for } \quad y \in \mathscr{G}
\end{gathered}
$$

where

$$
D(k)=\left(k^{2}+K^{2}\right) A(k) .
$$

Integration of (3.6) with respect to $y$ produces

$$
\int_{0}^{\infty} D(k) \cos k y d k= \begin{cases}C_{1} & \text { for } \quad a<y<b, \\ C_{2} & \text { for } c<y<\infty,\end{cases}
$$

where $C_{1}$ and $C_{2}$ are arbitrary constants.

Let

$$
\int_{0}^{\infty} D(k) \sin k y d k=h(y) \text { for } y \in \mathscr{W},
$$

where $h(y)$ is unknown. Then by Fourier inversion, (3.7) and (3.10) produce

$$
D(k)=\frac{2}{\pi} \int_{\mathscr{W}} h(t) \sin k t d t .
$$

Substitution of (3.11) in (3.9) gives

$$
\int_{\mathscr{W}} \frac{2 \operatorname{th}(t)}{t^{2}-y^{2}} d t= \begin{cases}\pi C_{1} & \text { for } a<y<b \\ \pi C_{2} & \text { for } c<y<\infty\end{cases}
$$


wherein the result

$$
\int_{0}^{\infty} \frac{\sin k t \sin k y}{k} d k=\frac{1}{2} \ln \left|\frac{y+t}{y-t}\right|
$$

has been utilized. Now, for consistency, $C_{2}$ in (3.12) must be taken to be zero, since the left side of (3.12) tends to zero as $y \rightarrow \infty$. Thus the multiple integral equations (3.6) and (3.7) reduce to a singular integral equation with a Cauchy kernel in a double interval, given by

$$
\int_{\mathscr{W}} \frac{2 \operatorname{th}(t)}{t^{2}-y^{2}} d t= \begin{cases}\pi C_{1} & \text { for } \quad a<y<b \\ 0 & \text { for } c<y<\infty\end{cases}
$$

The solution of (3.13) depends essentially on the behaviour of $h(t)$ near the end points $a, b, c$. To ascertain this behaviour, it is noted that for $y \in \mathscr{W}$

$$
\begin{aligned}
h(y) & =\int_{0}^{\infty}\left(k^{2}+K^{2}\right) A(k) \sin k y d k \\
& =-\left(\frac{d}{d y}+K\right)\left[\int_{0}^{\infty} A(k) L(k, y) d k-R e^{-K y}\right] \\
& =-\frac{1}{2}\left(\frac{d}{d y}+K\right)\{\phi(+0, y)-\phi(-0, y)\}
\end{aligned}
$$

so that $h(y)=-\left\{f^{\prime}(y)+K f(y)\right\} / 2$, where $f(y)$ is the difference of potential across the wall.

Thus, in view of (2.4),

$$
h(y)=O\left((y-d)^{1 / 2}\right) \quad \text { as } \quad y \rightarrow d,
$$

where $d$ represents any one of $a, b$ and $c$.

The solution of (3.13) satisfying (3.14) is given by

$$
h(t)= \begin{cases}-\frac{\lambda(t)}{\rho(t)} & \text { for } \quad a<t<b \\ \frac{\lambda(t)}{\rho(t)} & \text { for } \quad c<t<\infty\end{cases}
$$

(see Appendix), where

$$
\rho(t)=\left|\left(t^{2}-a^{2}\right)\left(b^{2}-t^{2}\right)\left(c^{2}-t^{2}\right)\right|^{1 / 2}
$$


and

$$
\lambda(t)=B_{0}+B_{1} t^{2}+\frac{2 C_{1}}{\pi} F(a, b, t)
$$

with

$$
F(a, b, t)=\int_{a}^{b} \frac{v \rho(v)}{v^{2}-t^{2}} d v
$$

and $B_{0}, B_{1}$ arbitrary constants determined below.

Substitution from (3.15) into (3.11) produces

$$
D(k)=\frac{2}{\pi}\left[-\int_{a}^{b} \frac{\lambda(y) \sin k y}{\rho(y)} d y+\int_{c}^{\infty} \frac{\lambda(y) \sin k y}{\rho(y)} d y\right] .
$$

By (3.8), $A(k)$ is now obtained in terms of the unknown constants $C_{1}, B_{0}, B_{1}$. To determine these constants, $A(k)$ is now substituted in (3.5). This gives after some manipulations

$$
R e^{-K y}=\left[-\int_{a}^{b} \frac{\lambda(u) e^{-K u}}{\rho(u)} d u+\int_{c}^{\infty} \frac{\lambda(u) e^{-K u}}{\rho(u)} d u\right] e^{-K y} \quad \text { for } \quad 0<y<a
$$

and

$$
R e^{-K y}=\left[-\int_{a}^{b} \frac{\lambda(u)}{\rho(u)}\left(e^{-K u}-e^{K u}\right) d u+\int_{c}^{\infty} \frac{\lambda(u) e^{-K u}}{\rho(u)} d u\right] e^{-K y} \quad \text { for } \quad b<y<c .
$$

Equations (3.20) and (3.21) produce two expressions for $R$, and as they must be the same, it is found that

$$
\int_{a}^{b} \frac{\lambda(u) e^{K u}}{\rho(u)} d u=0
$$

so that

$$
R=-\int_{a}^{b} \frac{\lambda(u) e^{-K u}}{\rho(u)} d u+\int_{c}^{\infty} \frac{\lambda(u) e^{-K u}}{\rho(u)} d u
$$

Equations (3.22) and (3.23) give two relations connecting the four unknowns $R, B_{0}, B_{1}$ and $C_{1}$. The other two relations can similarly be obtained by substituting $A(k)$ in (3.4). This gives after some simplification

$$
\begin{aligned}
i(1-R) e^{-K y}= & {\left[\int_{-a}^{a} \frac{\lambda(u) e^{-K u}}{\rho(u)} d u-\int_{b}^{c} \frac{\lambda(u) e^{-K u}}{\rho(u)} d u\right.} \\
& \left.-\frac{C_{1}}{K}\left(e^{-K a}-e^{K a}-e^{-K b}\right)\right] e^{-K y} \text { for } a<y<b
\end{aligned}
$$


and

$$
\begin{aligned}
i(1-R) e^{-K y}=[ & \int_{-a}^{a} \frac{\lambda(u) e^{-K u}}{\rho(u)} d u-\int_{b}^{c} \frac{\lambda(u)}{\rho(u)}\left(e^{-K u}+e^{K u}\right) d u \\
& \left.-\frac{C_{1}}{K}\left(e^{-K a}-e^{K a}-e^{-K b}+e^{K b}\right)\right] e^{-K y} \text { for } c<y<\infty .
\end{aligned}
$$

Now (3.24) and (3.25) produce two expressions for $i(1-R)$, and as they must be the same,

$$
\int_{b}^{c} \frac{\lambda(u) e^{K u}}{\rho(u)} d u+\frac{C_{1}}{K} e^{K b}=0
$$

so that

$$
i(1-R)=\int_{-a}^{a} \frac{\lambda(u) e^{-K u}}{\rho(u)} d u-\int_{b}^{c} \frac{\lambda(u) e^{-K u}}{\rho(u)} \frac{C_{1}}{K}\left(e^{-K a}-e^{K a}-e^{-K b}\right) d u .
$$

From the four relations (3.22), (3.23), (3.26) and (3.27), the four unknowns $R, B_{0}, B_{1}$ and $C_{1}$ can be calculated. Hence $\phi(x, y)$ can be fully determined from (3.1). The final expression for $R$ is given by

$$
R=i \frac{\delta_{1} A_{1}+\delta_{2} A_{2}-\frac{2}{\pi} A_{3}}{G_{3}-\delta_{1} G_{1}-\delta_{2} G_{2}}
$$

where

$$
\begin{aligned}
A_{1}= & \alpha_{2}(-K)-\alpha_{4}(-K), \quad A_{2}=\alpha_{2}^{\prime \prime}(-K)-\alpha_{4}^{\prime \prime}(-K) \\
A_{3}= & \alpha_{2}(-K, F)-\alpha_{4}(-K, F) \\
G_{1}= & \alpha_{1}(-K)-\alpha_{3}(-K)-i\left\{\alpha_{2}(-K)-\alpha_{4}(-K)\right\} \\
G_{2}= & \alpha_{1}^{\prime \prime}(-K)-\alpha_{3}^{\prime \prime}(-K)-i\left\{\alpha_{2}^{\prime \prime}(-K)-\alpha_{4}^{\prime \prime}(-K)\right\} \\
G_{3}= & \frac{2}{\pi}\left[\alpha_{1}(-K, F)-\alpha_{3}(-K, F)-i\left\{\alpha_{2}(-K, F)-\alpha_{4}(-K, F)\right\}\right] \\
& -\frac{1}{K}\left(e^{-K a}-e^{K a}-e^{-K b}\right) \\
\delta_{1}= & \frac{1}{\Delta}\left[\alpha_{2}^{\prime \prime}(K)\left\{\frac{e^{K b}}{K}+\frac{2}{\pi} \alpha_{3}(K, F)\right\}-\frac{2}{\pi} \alpha_{2}(K, F) \alpha_{3}^{\prime \prime}(K)\right] \\
\delta_{2}= & \frac{1}{\Delta}\left[\frac{2}{\pi} \alpha_{3}(K) \alpha_{2}(K, F)-\alpha_{2}(K)\left\{\frac{e^{K b}}{K}+\frac{2}{\pi} \alpha_{3}(K, F)\right\}\right] \\
\Delta= & \alpha_{3}(K) \alpha_{2}^{\prime \prime}(K)-\alpha_{3}^{\prime \prime}(K) \alpha_{2}(K)
\end{aligned}
$$




$$
\begin{aligned}
\alpha_{1}(K, F) & =\int_{-a}^{a} \frac{F(a, b, u) e^{K u}}{\rho(u)} d u, \quad \alpha_{2}(K, F)=\int_{a}^{b} \frac{F(a, b, u) e^{K u}}{\rho(u)} d u \\
\alpha_{3}(K, F) & =\int_{b}^{c} \frac{F(a, b, u) e^{K u}}{\rho(u)} d u, \quad \alpha_{4}(K, F)=\int_{c}^{\infty} \frac{F(a, b, u) e^{K u}}{\rho(u)} d u \\
\alpha_{i}(K) & \equiv \alpha_{i}(K, 1) \quad \text { and } \alpha_{i}^{\prime \prime}(K)=\frac{d^{2}}{d K^{2}} \alpha_{i}(K), i=1,2,3,4 .
\end{aligned}
$$

The expression for $R$ given in (3.28) coincides with that in [2] except for some signs in the expressions for $\Delta, \lambda_{1}$ and $\alpha_{4}(K, F)$.

(b) Method based on reduction to a singular integral equation with a combination of logarithmic and Cauchy kernels. If $g(y)$ denotes the horizontal component of velocity across the gaps, then it is easy to see that

$$
g(y)=O\left(|y-d|^{-1 / 2}\right) \quad \text { as } \quad y \rightarrow d,
$$

where $d$ is any of $a, b$ and $c$,

$$
\begin{aligned}
T & =1-R=-2 i \int_{\mathscr{G}} g(t) e^{-K t} d t \\
A(k) & =-B(k)=-\frac{2}{\pi} \frac{1}{k^{2}+K^{2}} \int_{\mathscr{G}} g(t) L(k, t) d t
\end{aligned}
$$

and $g(t)$ satisfies the following singular integral equation with a combination of logarithmic and Cauchy kernels in the double interval:

$$
\int_{\mathscr{G}} g(t)\left[K \ln \left|\frac{y-t}{y+t}\right|+\frac{1}{y-t}+\frac{1}{y+t}\right] d t=0 \quad \text { for } \quad y \in \mathscr{G}
$$

Also, for $y \in \mathscr{G}$,

$$
-\frac{\pi}{2} R e^{-K y}=\int_{0}^{\infty} \frac{L(k, y)}{k\left(k^{2}+K^{2}\right)}\left\{\int_{\mathscr{G}} g(t) L(k, t) d t\right\} d k .
$$

To solve the singular integral equation (3.35), let

$$
G(t)= \begin{cases}g(t) & \text { for } t \in \mathscr{G} \\ 0 & \text { for } t \in \mathscr{W}\end{cases}
$$

and

$$
H(t)= \begin{cases}0 & \text { for } t \in \mathscr{G} \\ h_{1}(t) & \text { for } t \in \mathscr{W}\end{cases}
$$


where $h_{1}(t)$ is unknown. Thus (3.35) is now equivalent to

$$
\int_{0}^{\infty} G(t)\left[K \ln \left|\frac{y-t}{y+t}\right|+\frac{1}{y-t}+\frac{1}{y+t}\right] d t=H(y) \quad \text { for } \quad 0<y<\infty .
$$

The function $h_{1}(t)$ has the behaviour

$$
h_{1}(t)=O\left(|t-d|^{-1 / 2}\right) \quad \text { as } \quad t \rightarrow d,
$$

where $d$ is any of $a, b$ and $c$.

The solution of (3.39) is given by (cf. [1])

$$
G(y)=\frac{d}{d y}\left[e^{-K y} \int_{0}^{y} e^{-K y} \int_{0}^{y} e^{K u}\left\{\frac{2}{\pi^{2}} \int_{0}^{\infty} \frac{t H(t)}{t^{2}-u^{2}} d t+S_{1}\right\} d u\right] \text { for } 0<y<\infty,
$$

where $S_{1}$ is an unknown constant and $H(t)$ involves the unknown function $h_{1}(t)$. An integral equation for $h_{1}(t)$ is obtained by observing that $G(y)=0$ for $y \in \mathscr{W}$ so that (3.41) gives

$$
\int_{0}^{y} e^{K u}\left[\frac{2}{\pi^{2}} \int_{0}^{\infty} \frac{t H(t)}{t^{2}-u^{2}} d t+S_{1}\right] d u= \begin{cases}S_{2} e^{K y} & \text { for } a<y<b, \\ S_{3} e^{K y} & \text { for } c<y<\infty,\end{cases}
$$

where $S_{2}, S_{3}$ are arbitrary constants. Noting (3.38), this produces

$$
\int_{\mathscr{W}} \frac{2 t h_{1}(t)}{t^{2}-y^{2}} d t= \begin{cases}C_{3} & \text { for } \quad a<y<b \\ C_{4} & \text { for } \quad c<y<\infty\end{cases}
$$

where

$$
\begin{aligned}
& C_{3}=\pi^{2} K S_{2}-S_{1}, \\
& C_{4}=\pi^{2} K S_{3}-S_{1},
\end{aligned}
$$

so that $C_{3}, C_{4}$ are arbitrary constants. However, for consistency we must have

$$
C_{4} \equiv 0
$$

since the left side of (3.42) tends to zero as $y \rightarrow \infty$. The solution of the singular integral equation (3.42) in the double interval $\mathscr{W}$ is given by

$$
h_{1}(y)= \begin{cases}-\frac{\lambda(y)}{\rho(y)} & \text { for } \quad a<y<b, \\ \frac{\lambda(y)}{\rho(y)} & \text { for } \quad c<y<\infty,\end{cases}
$$


(see Appendix), where

$$
\lambda(y)=\pi D_{0}+\pi D_{1} y^{2}+\frac{2 C_{3}}{\pi^{2}} F(a, b, y)
$$

with $\rho(y)$ and $F(a, b, y)$ being defined by (3.16) and (3.18), $D_{0}, D_{1}$ being unknown constants (the factor $\pi$ in $D_{0}, D_{1}$ being taken for convenience). Thus $\lambda(y)$ in (3.46) contains three arbitrary constants, $D_{0}, D_{1}$ and $C_{3}$. To determine these, we use the fact that $G(y)=0$ for $y \in \mathscr{W}$. This is equivalent to

$$
\frac{d}{d y}\left[e^{-K y} \frac{2}{\pi^{2}} \int_{0}^{y} e^{K u}\left(\int_{\mathscr{W}} \frac{t h_{1}(t)}{t^{2}-u^{2}} d t\right) d u-\frac{S_{1}}{K}\right]=0 \text { for } y \in \mathscr{W}
$$

For $a<y<b$, we obtain from (3.47) after using (3.42):

$$
\frac{d}{d y}\left[e^{-K y}\left\{\frac{2}{\pi^{2}} \int_{0}^{a} e^{K u}\left(\int_{\mathscr{W}} \frac{t h_{1}(t)}{t^{2}-u^{2}} d t\right) d u-\frac{C_{3}}{\pi^{2} K} e^{K a}-\frac{S_{1}}{K}\right\}\right]=0 .
$$

This produces

$$
\frac{1}{\pi^{2}} \int_{0}^{a} e^{K u}\left\{\int_{\mathscr{W}} \frac{t h_{1}(t)}{t^{2}-u^{2}} d t\right\} d u=\frac{C_{3} e^{K a}}{\pi^{2} K}+\frac{S_{1}}{K}
$$

Again, for $c<y<\infty$, we obtain from (3.47) after using (3.48) that

$$
\frac{d}{d y}\left[e^{-K y}\left\{\frac{C_{3} e^{K b}}{\pi^{2} K}+\frac{1}{\pi^{2}} \int_{b}^{c} e^{K u}\left(\int_{\mathscr{W}} \frac{2 t h_{1}(t)}{t^{2}-u^{2}} d t\right) d u\right\}\right]=0
$$

so that

$$
\int_{b}^{c} e^{K u}\left\{\int_{\mathscr{W}^{\prime}} \frac{2 t h_{1}(t)}{t^{2}-u^{2}} d t\right\} d u+\frac{C_{3} e^{K b}}{K}=0 .
$$

Finally, using (3.48) and (3.49), $g(y)$ is obtained from (3.41) as

$$
g(y)= \begin{cases}\frac{d}{d y}\left[e^{-K y}\left\{\frac{C_{0} e^{K u}}{\pi^{2} K}+\frac{1}{\pi^{2}} \int_{a}^{y} e^{K u}\left(\int_{\mathscr{W}} \frac{2 t h_{1}(t)}{t^{2}-u^{2}} d t\right) d u\right\}\right] & \text { for } 0<y<a, \\ \frac{d}{d y}\left[e^{-K y}\left\{\frac{1}{\pi^{2}} \int_{c}^{y} e^{K u}\left(\int_{\mathscr{W}} \frac{2 t h_{1}(t)}{t^{2}-u^{2}} d t\right) d u\right\}\right] & \text { for } b<y<c .\end{cases}
$$

Substitution of $h_{1}(t)$ from (3.45) into (3.50) and (3.49) produces after simplification

$$
g(y)= \begin{cases}\frac{d}{d y}\left[e^{-K y}\left\{\frac{C_{3} e^{K u}}{\pi^{2} K}-\int_{a}^{y} \frac{e^{K u} \lambda_{1}(u)}{\rho(u)} d u\right\}\right] & \text { for } 0<y<a, \\ \frac{d}{d y}\left[e^{-K y} \int_{c}^{y} \frac{e^{K u} \lambda_{1}(u)}{\rho(u)} d u\right] & \text { for } b<y<c\end{cases}
$$


with

$$
\begin{gathered}
\frac{C_{3} e^{K b}}{\pi^{2} K}+\int_{b}^{c} \frac{e^{K u} \lambda_{1}(u)}{\rho(u)} d u=0, \\
\lambda_{1}(u)=D_{0}+D_{1} u^{2}+\frac{2}{\pi} \frac{C_{3}}{\pi^{2}} F(a, b, u),
\end{gathered}
$$

where $\rho(u)$ and $F(a, b, u)$ are given in (3.16) and (3.18). Equation (3.53) gives a relation between the constants $D_{0}, D_{1}$ and $C_{3}$. The other relation connecting these constants can be obtained by substituting $g(y)$ from (3.51) and (3.52) into (3.36). It is found that

$$
\begin{aligned}
-R e^{-K y}=e^{-K y} & {\left[-\int_{a}^{b} \frac{\lambda_{1}(u)\left(e^{-K u}-e^{K u}\right)}{\rho(u)} d u\right.} \\
& \left.+\int_{c}^{\infty} \frac{\lambda_{1}(u) e^{-K u}}{\rho(u)} d u\right] \text { for } 0<y<a
\end{aligned}
$$

and

$$
-R e^{-K y}=e^{-K y}\left[-\int_{a}^{b} \frac{\lambda_{1}(u) e^{-K u}}{\rho(u)} d u+\int_{c}^{\infty} \frac{\lambda_{1}(u) e^{-K u}}{\rho(u)} d u\right] \text { for } b<y<c,
$$

so that

$$
\int_{a}^{b} \frac{\lambda_{1}(u) e^{K u}}{\rho(u)} d u=0
$$

and

$$
R=\int_{a}^{b} \frac{\lambda_{1}(u) e^{-K u}}{\rho(u)} d u-\int_{c}^{\infty} \frac{\lambda_{1}(u) e^{-K u}}{\rho(u)} d u .
$$

Again, substituting $g(y)$ from (3.51) and (3.52) into

$$
1-R=-2 i \int_{\mathscr{G}} g(t) e^{-K t} d t
$$

it is found that

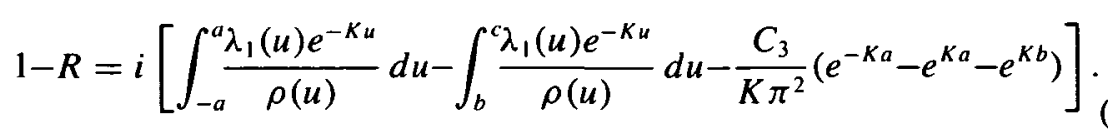

Now, (3.53), (3.55), (3.56) and (3.57) give four relations connecting the four constants $D_{0}, D_{1}, C_{3}$ and $R$, from which these can be calculated. It is verified that the expression for $R$ obtained here is exactly the same as given in (3.28). Finally, $\phi(x, y)$ can be determined from (3.1) after using $g(t)$ in (3.52), (3.53). 


\section{Conclusion}

The present paper demonstrates the use of two different integral equation formulations based on Havelock's expression of water wave potential to study the problem of water wave scattering by a completely submerged vertical wall with a gap. Explicit expressions for the reflection coefficient are obtained from both formulations.

\section{Appendix}

Here we obtain the solution of the singular integral equation

$$
\int_{\mathscr{W}} \frac{2 t p(t)}{t^{2}-p^{2}} d t= \begin{cases}M & \text { for } \quad a<y<b, \\ 0 & \text { for } c<y<\infty\end{cases}
$$

in the double interval $\mathscr{W}=(a, b) \cup(c, \infty)$ with the requirement that

$$
p(t)=O\left(|t-d|^{-1 / 2}\right) \quad \text { as } \quad t \rightarrow d,
$$

where $d$ is any of $a, b$ and $c$,

Substitution of $t^{2}=u, y^{2}=v$ reduces (A1) to

$$
\int_{\mathscr{W}_{1}} \frac{p_{1}(u)}{u-v} d u= \begin{cases}M & \text { for } \quad A<v<B, \\ 0 & \text { for } \quad C<v<\infty\end{cases}
$$

where

$$
A=a^{2}, B=b^{2}, C=c^{2}, p_{1}(u)=p\left(u^{1 / 2}\right), \mathscr{W}_{1}=(A, B) U(C, \infty) .
$$

Let

$$
w(z)=\frac{1}{2 \pi i} \int_{\mathscr{W}_{1}} \frac{p_{1}(u)}{u-z} d u, \quad z=x+i y .
$$

Use of the Plemelj formulae (cf. Muskhelishvili [5]) produces (after using (A3))

$$
\begin{array}{llll}
w^{+}(x)+w^{-}(x)=\frac{N(x)}{\pi i} & \text { for } & x \in \mathscr{W}_{1}, \\
w^{+}(x)-w^{-}(x)=p_{1}(x) & \text { for } & x \in \mathscr{W}_{1},
\end{array}
$$

where

$$
N(x)= \begin{cases}M & \text { for } \quad A<x<\infty \\ 0 & \text { for } \quad C<x<\infty\end{cases}
$$


and $w^{ \pm}(x)=\lim _{y \rightarrow \pm 0} w(z)$ for $x \in \mathscr{W}_{1}$. (A6) is a Riemann-Hilbert problem for the sectionally analytic function $w(z)$ in the complex $z$-plane cut along the real axis from $A$ to $B$ and from $C$ to $\infty$. Its solution is given by

$$
w(z)=w_{0}(z)\left[\frac{P}{2}+\frac{Q z}{2}+\frac{1}{2 \pi i} \int_{\mathscr{W}_{1}} \frac{N(u)}{\pi i w_{0}^{+}(u)} \frac{d u}{u-z}\right]
$$

(cf. [5]), where

$$
w_{0}(z)=\{(z-a)(z-b)(z-c)\}^{1 / 2}
$$

and $P, Q$ are arbitrary constants, $w_{0}^{+}(x)=\lim _{y \rightarrow+0} w_{0}(z), x \in \mathscr{W}_{1}$.

Applying the Plemelj formula to $w(z)$ in (A9) and using the relation $\mathrm{A}(7)$, we obtain $p_{1}(x)$ and hence $p(t)$ by (A4). Then $p(x)$ is obtained as

$$
p(x)= \begin{cases}-\frac{\lambda_{0}(x)}{\rho(x)} & \text { for } \quad a<x<b, \\ \frac{\lambda_{0}(x)}{\rho(x)} & \text { for } \quad c<x<\infty\end{cases}
$$

where

$$
\lambda_{0}(x)=P+Q x^{2}+\frac{2 N}{\pi^{2}} F(a, b, x),
$$

$\rho(x)$ and $F(a, b, x)$ being defined by (3.16) and (3.18).

\section{Acknowledgement}

'This work is partially supported by DST, New Delhi, through a research project with Sudeshna Banerjea and by CSIR, New Delhi, through a research project with B. N. Mandal.

\section{References}

[1] Sudeshna Banerjea and B. N. Mandal, "Solution of a singular integral equation in a double interval arising in the theory of water waves", Appl. Math. Lett. 6 (1993) 81-84.

[2] A. Chakrabarti and L. Vijayabharati, "Transmission of water waves through a gap in a submerged vertical barrier", Indian J. Pure Appl. Math. 22 (1991) 491-512.

[3] W. R. Dean, "On the reflection of surface waves by a submerged plane barrier", Proc. Camb. Phil. Soc. 41 (1945) 231-238. 
[4] D. V. Evans, "Diffraction of water waves by a submerged vertical plate", J. Fluid Mech. 40 (1970) 433-451.

[5] N. I. Muskhelishvili, Singular integral equations (Noordhoff, Groningen, 1963).

[6] D. Porter, "The transmission of surface waves through a gap in a vertical barrier", Proc. Camb. Phil. Soc. 71 (1972) $411-422$.

[7] F. Ursell, "The effect of a fixed vertical barrier on surface waves in deep water", Proc. Camb. Phil. Soc. 43 (1947) 374-382. 